ISSN 2179-6750

\title{
Atenção à saúde maternoinfantil e saúde bucal nos ciclos do PMAQ-AB
}

Attention to mother and child health and oral health at PMAQ-AB cycles Atención de salud materno-infantily de salud bucal en los ciclos de PMAQ-AB

\author{
Suzely Adas Saliba MOIMAZ (1) \\ Tânia Adas SALIBA ${ }^{(2)}$ \\ Renato Moreira ARCIERI ${ }^{(2)}$ \\ Léa LOFEGO ${ }^{(3)}$ \\ Nemre Adas SALIBA ${ }^{(1)}$
}

Recebido: 8 out 2016 Revisado: 15 mar 2017 Aceito: 01 abr 2017

\section{Autor de}

correspondência: Suzely Adas Saliba Moimaz

sasaliba@foa.unesp.br

Conflito de interesses Os autores declaram não haver nenhum interesse profissional ou pessoal que possa gerar conflito de interesses em relação a este manuscrito.

\section{Resumo}

As ações de Vigilância em Saúde são essenciais para se garantir a resolutividade da assistência e aplicabilidade dos princípios e diretrizes do Sistema único de Saúde. O objetivo nesta pesquisa foi avaliar o acesso e a qualidade dos serviços de saúde maternoinfantil e saúde bucal de um município do estado de São Paulo. Realizou-se uma pesquisa descritiva, de caráter exploratório, com uma abordagem quantitativa dos dados do Programa Nacional de Melhoria do Acesso e Qualidade da Atenção Básica. Foram analisadas as seguintes variáveis: Atendimentos de pré-natal por gestante cadastrada; Consultas de puericultura por criança cadastrada; Crianças menores de 04 meses com aleitamento exclusivo; Crianças menores de 01 ano com vacina em dia; Ação coletiva de escovação dental supervisionada; primeira consulta odontológica programática; primeira consulta de atendimento odontológico à gestante; Razão entre tratamentos concluídos e primeiras consultas odontológicas programáticas. O município apresentou média de atendimentos de pré-natal de 5,79; média de consultas por criança de 0,48; proporção de $96,30 \%$ das crianças com aleitamento exclusivo e $100 \%$ com vacina em dia. Quanto à saúde bucal a cobertura de escovação supervisionada foi de 2,33\%; de primeira consulta programática de $0,73 \%$; primeira consulta à gestante de 3,85\%. A taxa de resolutividade foi de $12,22 \%$. Conclui-se que o acesso e a qualidade das ações e serviços em saúde foram garantidos, entretanto, especificamente na área de saúde bucal é necessário ampliar o acesso às gestantes e aumentar a resolutividade dos serviços prestados.

Descritores: Saúde materno-infantil; Saúde Bucal; Qualidade, Acesso e Avaliação da Assistência à Saúde. 


\begin{abstract}
Health surveillance actions are essential to guarantee health care outcomes and applicability of principles and guidelines of the Brazilian Unified Health System. The purpose of this study was to evaluate access and quality of maternal and child health services and oral health in a city in São Paulo state. A descriptive and exploratory study was performed with a quantitative approach to data of the National Program for Access and Quality Improvement in Primary Care. The following variables were analyzed: prenatal visits per enrolled pregnant woman; childcare visits per enrolled child; exclusively breastfed children under four months old; children under one year old with updated mandatory immunization records; collective action for supervised toothbrushing; first scheduled dental visit; first dental care visit for pregnant women; ratio between completed treatments and first scheduled dental visits. The city had an average of 5.79 prenatal care visits; 0.48 visits per child; $96.30 \%$ exclusively breastfed children and $100 \%$ children with updated immunization records. Concerning oral health, supervised brushing coverage was $2.33 \%$; first scheduled visit was $0.73 \%$; first visit for pregnant women was $3.85 \%$. The outcome rate was $12.22 \%$. It was concluded that access and quality of health services and activities have been assured, however, it is imperative to expand access to pregnant women and increase the outcome of services provided for oral health.
\end{abstract}

Keywords: Maternal and child health; Oral Health; Quality, Access and Evaluation of Health Care.

\begin{abstract}
Resumen
Las acciones de vigilancia en salud son esenciales para garantizar la resolución de la asistencia y aplicación de los principios y directrices del Sistema Único de Salud. El objetivo en esta investigación fue evaluar el acceso y la calidad de los servicios de salud materno-infantil y de salud bucal de un municipio del estado de Sao Paulo. Se realizó una investigación descriptiva, de carácter exploratorio, con un enfoque cuantitativo de los datos del Programa Nacional de Mejoría del Acceso y Calidad de la Atención Básica. Fueron analizadas las siguientes variables: Atención prenatal por gestante registrada; Consultas de puericultura por niño registrado; Niños menores de 04 meses con lactancia exclusiva; Niños menores de 01 año con vacuna al día; Acción colectiva de cepillado dental supervisado; Primera consulta odontológica programática ; Primera consulta de atención odontológica a la gestante; Relación entre tratamientos concluidos y primeras consultas odontológicas programáticas. El municipio presentó media de atención pre-natal de 5,79; media de consultas por niño de 0,48 ; proporción de $96,30 \%$ de los niños con lactancia exclusiva y $100 \%$ con vacuna al día. En cuanto a la salud bucal la cobertura de cepillado supervisado fue de 2,33\%; de primera consulta programática de $0,73 \%$; primera consulta de la gestante de $3,85 \%$. La tasa de resolución fue de $12,22 \%$. Se concluye que el acceso y la calidad de las acciones y servicios en salud fueron garantizados, entretanto, específicamente en el área de salud bucal es necesario ampliar el acceso a las gestantes y aumentar la resolución de los servicios prestados.
\end{abstract}

Palabras-claves: Salud materno-infantil; Salud Bucal; Calidad, Acceso y Evaluación de la Asistencia de Salud.

\title{
Introdução
}

Historicamente, o Brasil é marcado por um modelo de atenção à saúde excludente e assistencialista. A promulgação da Constituição da República Federativa do Brasil, em 1988, inicia as mudanças na área da saúde com a garantia a todo cidadão brasileiro ao 
acesso integral, universal e gratuito a serviços de saúde. Neste contexto, em 1990 o Sistema Único de Saúde (SUS) é instituído ${ }^{1}$ e emerge como uma conquista de movimentos sociais que reivindicavam mudanças no modelo de atenção à saúde.

A transição de uma atenção centrada na doença para um modelo de cuidado integral em saúde evidencia o grande avanço nas Políticas Públicas Nacionais, ${ }^{2,3}$ entretanto, a garantia e a aplicabilidade dos princípios e diretrizes do SUS, em um país caracterizado por diferentes contextos regionais é uma tarefa extremamente difícil.

Várias e constantes são as tentativas de ampliação de mecanismos que levem à otimização dos serviços públicos de saúde e ao acesso dos usuários a todos os níveis de atenção do sistema, entretanto, a questão assume uma forte complexidade ao considerarem-se as enormes desigualdades geográficas, culturais e socioeconômicas que há tempos permeiam o país. ${ }^{4}$

Em razão da heterogeneidade e da dinamicidade da saúde, o SUS está permanentemente se reorganizando através de instrumentos de gestão como o Pacto pela Saúde, ${ }^{5}$ Programa Nacional de Avaliação de Serviços de Saúde (PNASS), ${ }^{6}$ Programa de Melhoria do Acesso e Qualidade da Atenção Básica (PMAQ-AB). ${ }^{7}$

O PMAQ-AB foi lançado em 2011 com o objetivo de melhorar a qualidade e o acesso aos serviços de saúde oferecidos à população, assim como, favorecer a ocorrência de mudanças no nível local de forma rápida e objetiva, buscando um padrão de qualidade comparável nacional, regional e municipalmente. ${ }^{8} \mathrm{O}$ programa propõe o desenvolvimento de um conjunto de ações, como: autoavaliação; apoio institucional; monitoramento e avaliação dos indicadores de saúde; educação permanente e cooperação horizontal. A execução destas atividades é extremamente relevante para a eficácia, eficiência e efetividade das políticas públicas de saúde, embora, ainda hoje, a grande maioria dos municípios brasileiros não está estruturada para o seu desenvolvimento.

As ações de Vigilância em Saúde como o monitoramento e a avaliação constituemse em ferramentas para aprimorar o sistema, pois permitem o conhecimento situacional, 
informam resultados, apontam necessidades de mudanças, ${ }^{9}$ proporcionam subsídios para a organização e fortalecimento do SUS, principalmente no que se refere à Atenção Primária em Saúde (APS) na medida em que representa a principal porta de entrada dos usuários a todos os níveis de cuidado: primário, secundário e terciário. ${ }^{10}$ Como exemplo prático, a Política Nacional de Atenção Básica regulamenta a participação no planejamento local de saúde, no monitoramento e avaliação das ações da equipe, unidade e município, visando à readequação do processo de trabalho e do planejamento diante das necessidades, realidade, dificuldades e possibilidades analisadas. ${ }^{2}$

A Atenção Básica caracteriza-se por um conjunto de ações de saúde, no âmbito individual e coletivo, que abrange a promoção e a proteção da saúde, a prevenção de agravos, o diagnóstico, o tratamento, a reabilitação, redução de danos e a manutenção da saúde com o objetivo de desenvolver uma atenção integral que impacte na situação de saúde e autonomia das pessoas e nos determinantes e condicionantes de saúde das coletividades. $^{2}$ Este nível de cuidado tem capacidade de resolução de $80 \%$ dos problemas de saúde. Neste amplo contexto de atuação da atenção primária em saúde são elencadas algumas áreas estratégicas como a saúde da mulher, da criança e a saúde bucal, para as quais, Políticas Públicas foram instituídas ${ }^{11-13}$ visando a ampliação do acesso, da qualificação e da humanização dos serviços, a redução da morbimortalidade feminina, materna e infantil; a redução da prevalência e incidência dos problemas bucais.

Em estudo realizado na Austrália por membros da política, da academia, da prática clínica e consumidores para avaliação dos serviços de Atenção Primária à Saúde que deveriam estar à disposição de todos os australianos, independente do local de moradia, foram citados com forte consenso, os serviços de saúde maternoinfantil, saúde bucal, prevenção em saúde, entre outros. ${ }^{14}$

A redução da mortalidade infantil e a melhora da saúde das gestantes foram elencadas como metas do milênio pela Organização das Nações Unidas (ONU), evidenciando a relevância e a necessidade de priorização do cuidado maternoinfantil. 
Considerando que as ações de vigilância em saúde como o monitoramento e avaliação dos indicadores de saúde maternoinfantil e de saúde bucal são essenciais para a resolutividade da assistência e para a melhoria da qualidade de vida da população, realizou-se esse trabalho.

\section{Objetivo}

O objetivo nesta pesquisa foi avaliar o acesso e a qualidade das ações e serviços de saúde maternoinfantil e saúde bucal de um município do estado de São Paulo, a partir de dados obtidos da certificação do Programa Nacional de Melhoria do Acesso e Qualidade da Atenção Básica.

\section{Metodologia}

Trata-se de uma pesquisa descritiva, de caráter exploratório com abordagem quantitativa.

Foram consultados os relatórios do Programa Nacional de Melhoria do Acesso e Qualidade da Atenção Básica e verificados os dados secundários no site do Ministério da Saúde em http://dabsistemas.saude.gov.br/sistemas/sgdab/login.php para as certificações do $1^{\circ}$ e $2^{\circ}$ ciclo de um município do estado de São Paulo.

Os indicadores das linhas prioritárias, maternoinfantil e saúde bucal, foram verificados e as seguintes variáveis analisadas.

Atenção à saúde materna:

1. Proporção de gestantes cadastradas (sobre as estimadas);

2. Número médio de atendimentos de pré-natal por gestante cadastrada;

3. Proporção de gestantes que iniciaram o pré-natal no primeiro trimestre;

4. Proporção de gestantes com pré-natal em dia;

5. Proporção de gestantes com vacina em dia.

Atenção à saúde infantil: 
1. Média de consultas de puericultura por criança cadastrada;

2. Proporção de crianças menores de 04 meses com aleitamento exclusivo;

3. Proporção de crianças menores de 01 ano com vacina em dia;

4. Proporção de crianças menores de 02 anos pesadas;

5. Média de consultas médicas para menores de 01 ano;

6. Média de consultas médicas para menores de 05 anos.

Atenção à saúde bucal:

1. Média da ação coletiva de escovação dental supervisionada;

2. Cobertura de primeira odontológica programática;

3. Cobertura de $1^{\mathrm{a}}$ consulta de atendimento odontológico à gestante;

4. Razão entre tratamentos concluídos e primeiras consultas odontológicas programáticas.

Os resultados dos indicadores foram analisados de acordo com os parâmetros estabelecidos pela Nota Metodológica publicada pelo Ministério da Saúde ${ }^{15}$, comparados com Extrato, Estado e Brasil e entre $1^{\circ}$ e $2^{\circ}$ ciclo do programa.

\section{Resultados e discussões}

Os resultados referentes ao município, ao estrato, ao estado e ao Brasil no $2^{\circ}$ ciclo do programa são apresentados na Tabela 1.

Tabela 1. Comparativo dos indicadores da atenção à saúde maternoinfantil e saúde bucal

\begin{tabular}{|c|c|c|c|c|}
\hline \multirow{2}{*}{$\begin{array}{c}\text { Indicadores saúde maternoinfantil } \\
\text { e saúde bucal }\end{array}$} & \multicolumn{4}{|c|}{$\begin{array}{l}\text { Resultados } 2^{\circ} \text { Ciclo } \\
\text { Local }\end{array}$} \\
\hline & Município & Estrato & Estado & Brasil \\
\hline $\begin{array}{l}\text { Proporção de gestantes cadastradas (sobre as } \\
\text { estimadas) }\end{array}$ & 98,05 & 55,11 & 66,78 & 52,25 \\
\hline $\begin{array}{l}\text { Número médio de atendimentos de pré-natal por } \\
\text { gestante cadastrada }\end{array}$ & 5,79 & 7,00 & 6,66 & 7,14 \\
\hline $\begin{array}{l}\text { Proporção de gestantes que iniciaram o pré-natal } \\
\text { no primeiro trimestre }\end{array}$ & 93,83 & 87,60 & 87,76 & 83,73 \\
\hline
\end{tabular}


ISSN 2179-6750

\begin{tabular}{|c|c|c|c|c|}
\hline Proporção de gestantes com pré-natal em dia & 100,00 & 95,76 & 92,26 & 92,00 \\
\hline Proporção de gestantes com vacina em dia & 100,00 & 96,92 & 92,80 & 93,67 \\
\hline $\begin{array}{l}\text { Média de consultas de puericultura por criança } \\
\text { cadastrada }\end{array}$ & 0,48 & 5,74 & 5,26 & 5,24 \\
\hline $\begin{array}{l}\text { Proporção de crianças menores de } 04 \text { meses com } \\
\text { aleitamento exclusivo }\end{array}$ & 96,30 & 74,55 & 76,22 & 73,76 \\
\hline $\begin{array}{l}\text { Proporção de crianças menores de } 01 \text { ano com } \\
\text { vacina em dia }\end{array}$ & 100,00 & 98,01 & 96,60 & 95,42 \\
\hline $\begin{array}{l}\text { Proporção de crianças menores de } 02 \text { anos } \\
\text { pesadas }\end{array}$ & 81,39 & 92,95 & 90,51 & 87,24 \\
\hline $\begin{array}{l}\text { Média de consultas médicas para menores de } 01 \\
\text { ano }\end{array}$ & 19,47 & 4,52 & 4,77 & 3,75 \\
\hline $\begin{array}{l}\text { Média de consultas médicas para menores de } 05 \\
\text { anos }\end{array}$ & 14,42 & 3,06 & 2,12 & 2,23 \\
\hline $\begin{array}{l}\text { Média da ação coletiva escovação dental } \\
\text { supervisionada }\end{array}$ & 2,33 & 3,90 & 2,06 & 2,48 \\
\hline $\begin{array}{l}\text { Cobertura de primeira consulta odontológica } \\
\text { programática }\end{array}$ & 0,73 & 1,43 & 0,64 & 1,00 \\
\hline $\begin{array}{l}\text { Cobertura de } 1^{\text {a }} \text { consulta de atendimento } \\
\text { odontológico à gestante }\end{array}$ & 3,85 & 15,26 & 9,41 & 11,67 \\
\hline $\begin{array}{l}\text { Razão entre tratamentos concluídos e primeiras } \\
\text { consultas odontológicas programáticas }\end{array}$ & 12,22 & 43,89 & 65,37 & 45,27 \\
\hline
\end{tabular}

Fonte: Relatório descritivo PMAQ-AB (2015).

Nesta pesquisa sobre os resultados do PMAQ-AB notou-se que o município está garantindo atendimento e acompanhamento adequados durante a gestação, importante fator para a redução da morbimortalidade materna e infantil. Dentre os 5 indicadores analisados relacionados à saúde materna, apenas o número médio de atendimentos de pré-natal por gestante cadastrada obteve resultado inferior quando comparado ao estrato, estado e ao Brasil, embora, essa diferença não tenha sido significativa e atenda ao preconizado pelo Ministério da Saúde, que recomenda minimamente, seis consultas de pré-natal. ${ }^{16}$

O Gráfico 1 apresenta os resultados da saúde materna no $1^{\circ}$ e $2^{\circ}$ ciclo do programa. 


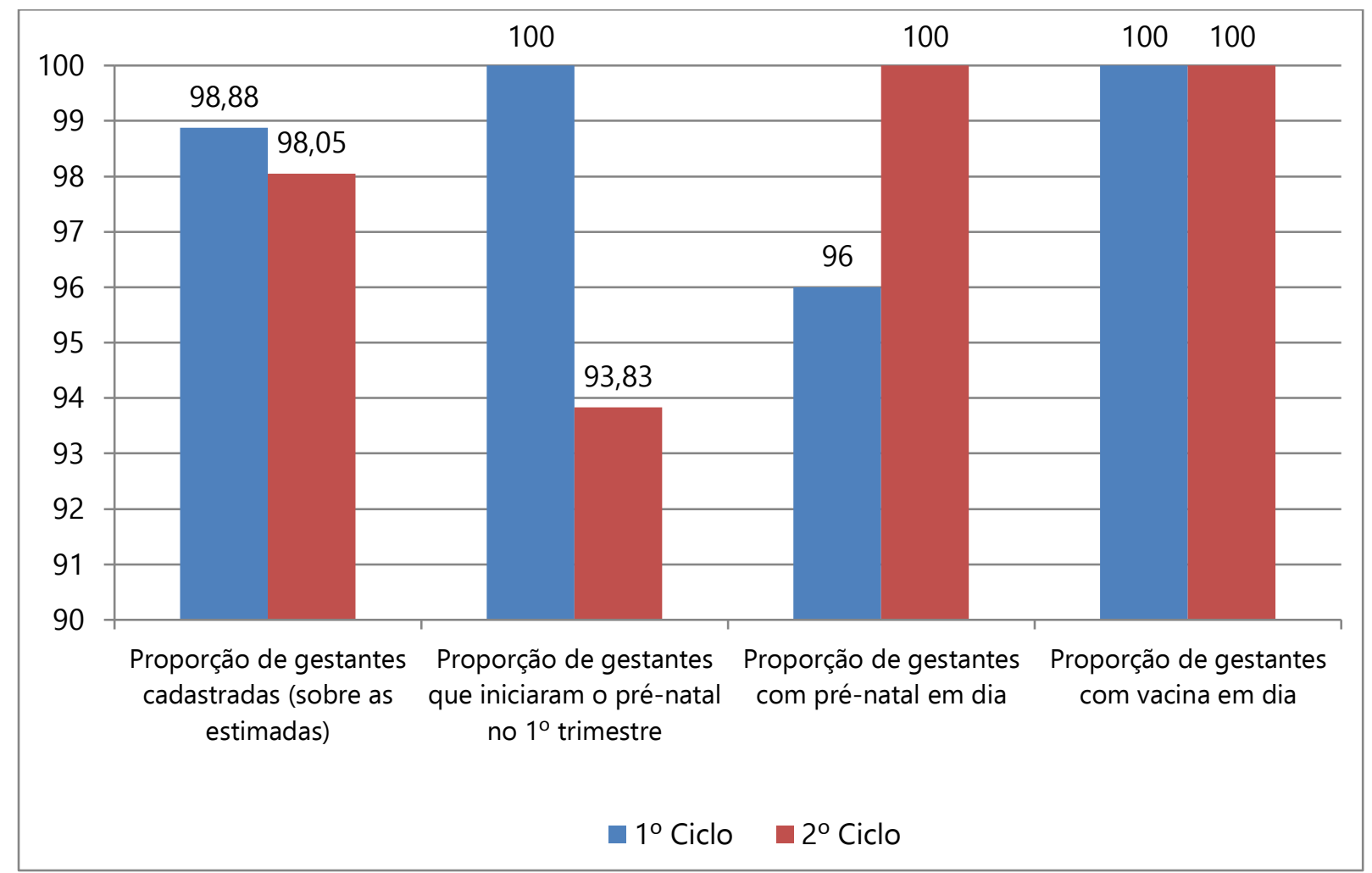

Gráfico 1. Indicadores municipais da atenção à saúde materna.

Fonte: Relatório descritivo PMAQ-AB (2015).

A análise comparativa dos indicadores sinaliza fragmentações na prestação dos serviços, caracterizada pela descontinuidade das ações no pré-natal entre o primeiro e o segundo ciclo do programa. O indicador proporção de gestantes que iniciaram o pré-natal no primeiro trimestre apresentou uma queda de $6,17 \%$ e o número médio de atendimentos de pré-natal por gestante cadastrada reduziu de 9,94 para 5,79.

Costa observou no estado do Maranhão que menos da metade das gestantes que realizou pré-natal, o fez de forma adequada, ou seja, iniciou o pré-natal no primeiro trimestre com no mínimo seis consultas para uma gestação a termo. ${ }^{17}$

Apesar da queda nos resultados dos indicadores no presente estudo e do baixo percentual de gestantes que realizaram pré-natal de maneira adequada no estado do Maranhão, ${ }^{17}$ é sabido que os parâmetros são exequíveis, neste sentido, evidencia-se a necessidade da adoção de condutas periódicas de vigilância em saúde para a qualificação da assistência pré-natal. 
Embora o pré-natal seja considerado um dos quatro pilares da maternidade segura, poucas avaliações têm sido realizadas sobre a sua realidade, a fim de determinar quais intervenções teriam melhores resultados. ${ }^{18}$ Moimaz $^{19}$ refere ser necessário o acompanhamento e avaliação permanente dos sistemas de informação em saúde, para que melhorias possam ser introduzidas e falhas corrigidas. ${ }^{19}$ Outro instrumento de grande valia para a organização do processo de trabalho e qualificação da assistência pré-natal é a gestão compartilhada, onde toda a equipe de saúde participa do planejamento, da avaliação, de discussões sobre as dificuldades enfrentadas e do processo de pactuação de metas.

No que tange a atenção à saúde infantil, o indicador, média de consultas de puericultura por criança cadastrada apresentou resultado muito aquém em relação ao estrato, estado e ao Brasil, além de ausência de produção no ciclo anterior. Os resultados da atenção à saúde infantil estão relacionados na Tabela 1, nos Gráficos 2 e 3.

Ao analisar os indicadores, média de consultas médicas para menores de um e cinco anos observa-se discrepância nos valores em relação às referências, sendo, respectivamente, cinco e seis vezes maiores que a média nacional. O elevado valor no número médio destas consultas indica que a assistência médica e de enfermagem em puericultura tenha ocorrido, sendo provável a existência de problemas de registros e informação. 


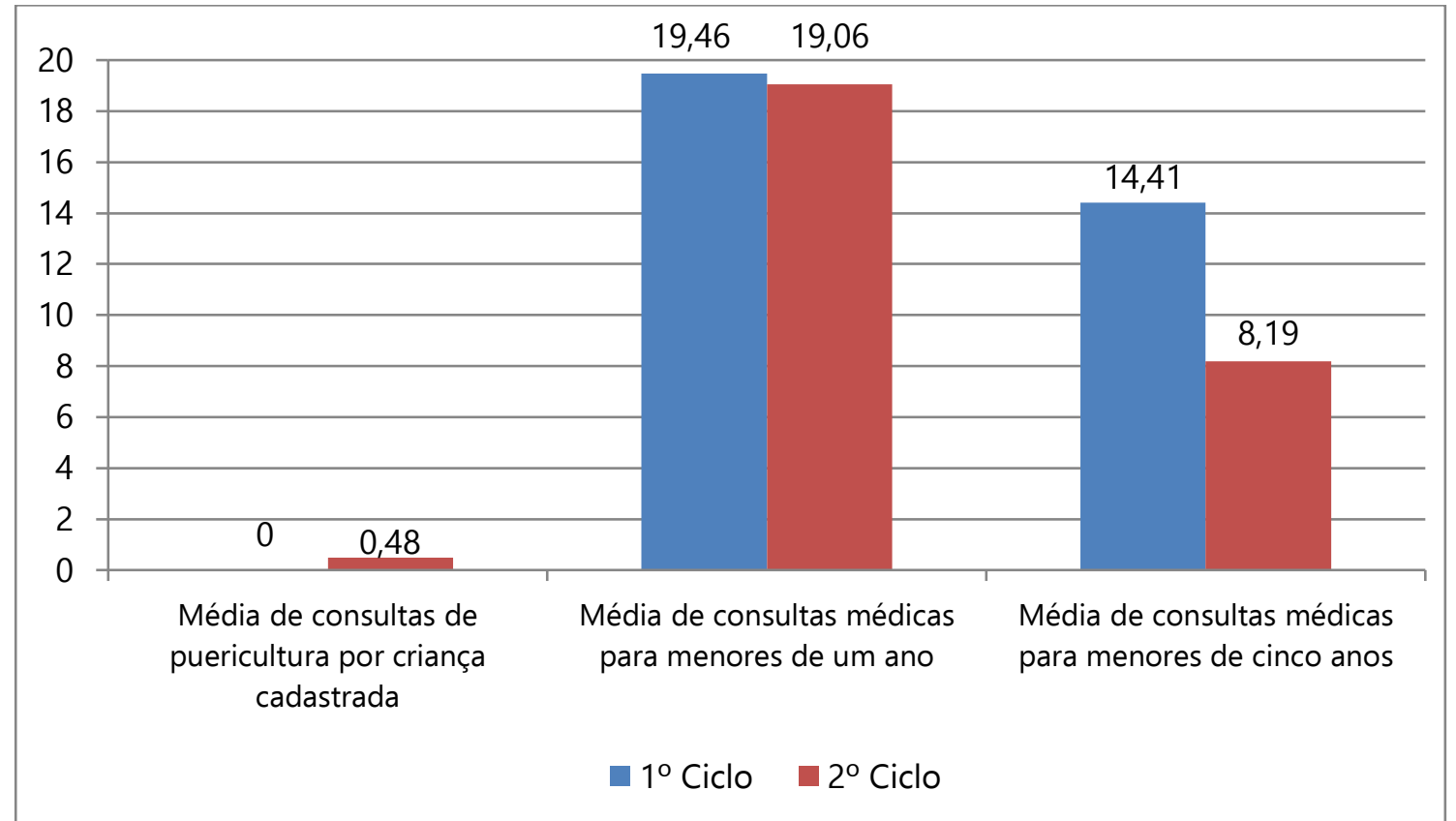

Gráfico 2. Indicadores municipais da atenção à saúde infantil no $1^{\circ}$ e $2^{\circ}$ ciclo. Fonte: Relatório descritivo PMAQ-AB (2015).

A ausência de capacitações e qualificação profissional, a não compreensão da importância da informação em saúde, a falta de cultura de uso do dado para planejamento e avaliação dos serviços, a demanda excessiva de atividades laborais e a escassez de recursos são fatores que contribuem para a utilização de códigos errados no registro de procedimentos, para a não alimentação dos sistemas de informação e para a ocorrência de inconsistências prejudicando a qualidade da informação.

A importância do registro de dados do serviço deve ser trabalhada com toda a equipe de saúde a fim de garantir a autenticidade e confiabilidade da informação, essenciais para a gestão da saúde pública.

Quanto às práticas de promoção e prevenção da saúde maternoinfantil como o incentivo ao aleitamento materno e a vacinação, pode-se observar que estão sendo efetivas, já que a proporção de crianças menores de 04 meses com aleitamento materno exclusivo aumentou $32,09 \%$ de um ciclo para o outro e a vacinação de crianças menores de um ano e gestantes mantiveram com $100 \%$ de cobertura em ambos os ciclos, 
evidenciando a valorização e o esforço da equipe de saúde para a conscientização da população através de atividades educativas, busca ativa, trabalho multidisciplinar, intersetorial e coordenação do cuidado. Esses dados podem ser verificados no Gráfico 3.

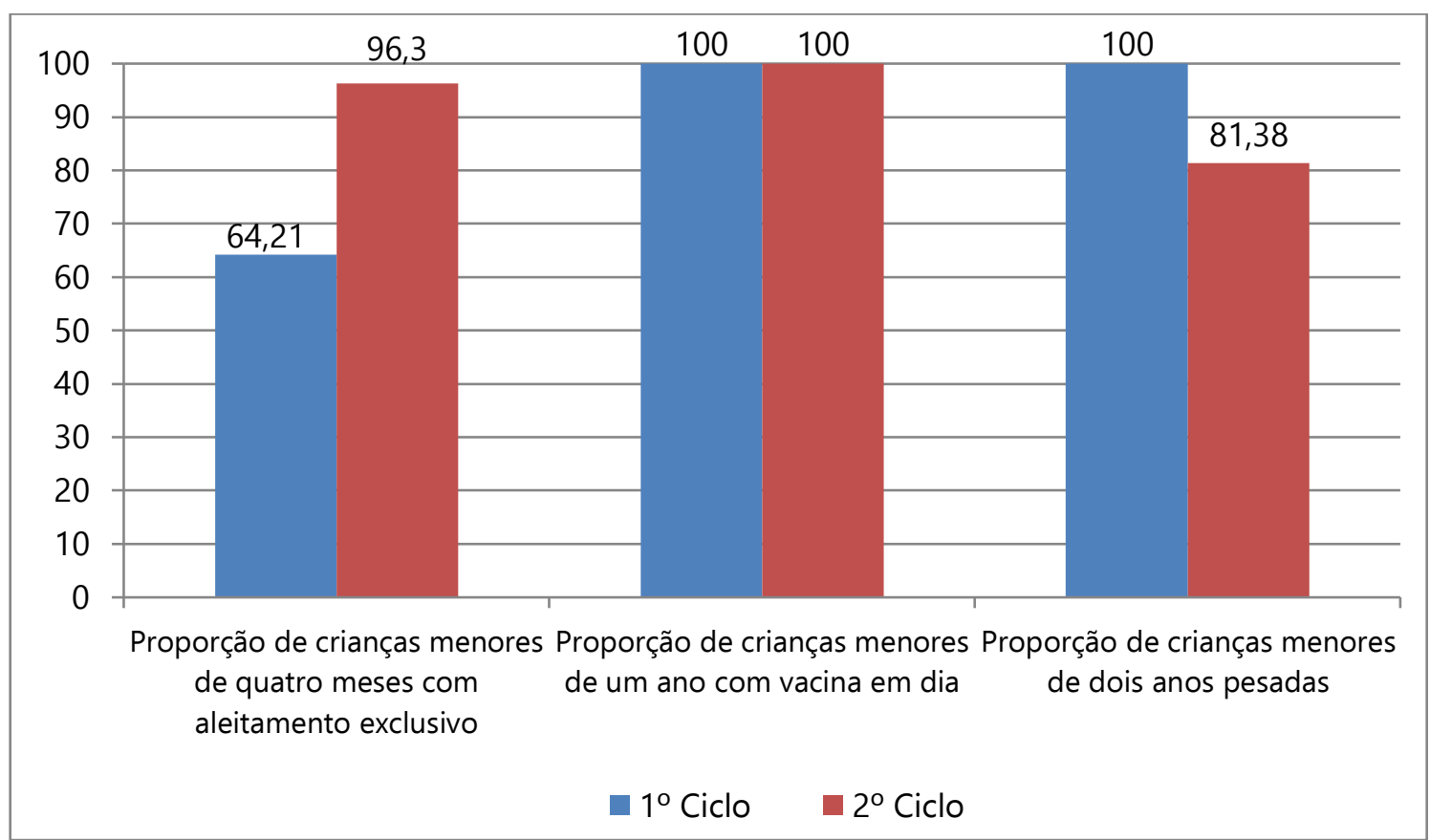

Gráfico 3. Indicadores municipais da atenção à saúde infantil no $1^{\circ}$ e $2^{\circ}$ ciclo.

Fonte: Relatório descritivo PMAQ-AB (2015).

Apesar do bom desempenho e crescente melhora observados neste estudo quanto à adoção da amamentação exclusiva, há muitas evidências na literatura de dificuldades na efetivação desta ação. Em um estudo realizado em escolas municipais de educação infantil observou - se que $66,6 \%$ das mães não amamentaram exclusivamente seus filhos até o sexto mês de vida. ${ }^{20}$ Outra pesquisa aponta que já no primeiro mês, muitas mães complementaram a alimentação de seu filho e que nenhuma criança aos seis meses foi amamentada exclusivamente no peito pelas mães. ${ }^{21}$

Perante a diversidade organizacional e estrutural da atenção à saúde infantil no Brasil e da relevância do cuidado, já que este permite o diagnóstico precoce de problemas 
relacionados ao crescimento, estado nutricional, desenvolvimento neuropsicomotor e comportamental, além da prevenção de doenças imunopreviníveis e promoção de hábitos de vida saudáveis ${ }^{15}$ é necessário intensificar o acompanhamento das crianças nas Unidades de Saúde e o monitoramento dos indicadores como fator preponderante para a qualidade na assistência à saúde infantil. ${ }^{22}$

As equipes de saúde, principalmente as equipes de Atenção Básica/Estratégia Saúde da Família desenvolvem atividades educativas na perspectiva da valorização do autocuidado, da conscientização do usuário pela responsabilidade com a própria saúde, da sua família e da coletividade. O grupo de gestantes, por exemplo, é uma atividade realizada por multiprofissionais que orientam, cada um na sua especialidade, sobre os cuidados na gestação e no período puerperal.

Ressalta-se, na atenção à saúde infantil, a relevância do papel da família para o desenvolvimento da criança. Trata-se de uma fase da vida que merece atenção envolvendo os demais integrantes da família. ${ }^{23}$ Os profissionais de saúde devem estar atentos para a integração e articulação entre cuidador responsável, equipe de saúde e família.

Na área da saúde bucal, os indicadores, média da ação coletiva de escovação dental supervisionada e cobertura de primeira consulta odontológica programática, apresentaram valores abaixo da média nacional, porém acima do estado e do estrato, respectivamente. Já os indicadores cobertura de primeira consulta de atendimento odontológico à gestante e razão entre tratamentos concluídos e primeiras consultas odontológicas programáticas, houve grande variação em relação às referencias, sendo o primeiro $11,41 \%$ aquém do estrato e o segundo $53,15 \%$ inferior ao estado. Esses dados estão descritos na Tabela 1.

A avaliação dos indicadores de saúde bucal evidencia baixa resolutividade dos serviços e acesso prejudicado das gestantes apontando para a necessidade reorganização do processo de trabalho, da agenda dos profissionais e busca ativa das gestantes a fim de priorizar o cuidado continuado, facilitar e incentivar o retorno do paciente para a 
conclusão do tratamento e garantir o pré-natal odontológico.

Em revisão da literatura sobre o pré-natal odontológico foi verificado que apesar dos avanços na odontologia, muitos cirurgiões-dentistas ainda possuem a concepção de que o tratamento odontológico à gestante deve ser postergado e que procedimentos como tomadas radiográficas e anestesias não devem ser realizados. Entretanto sabe-se que neste período a gestante constitui um grupo de risco uma vez que as mudanças hormonais, físicas e psicológicas podem desencadear um desequilíbrio à saúde bucal da mulher. ${ }^{24}$

A implantação do plano de carreira, que constitui incentivo à qualificação profissional, e o apoio institucional à capacitação dos servidores através do aperfeiçoamento, especialização, atividades de educação permanente e continuada são ferramentas organizacionais que contribuem para a melhoria na prestação dos serviços, mudança no cenário odontologia/gestação e consequentemente impactam positivamente na qualidade de vida da população e na satisfação do usuário.

O atendimento odontológico às gestantes é seguro e apresenta muitos benefícios, tanto para a mãe quanto para o bebê. ${ }^{24}$

Deficiências, como as encontradas neste estudo, também foram relatadas por Oliveira et al que observam baixa resolutividade da atenção odontológica e concluíram que a implantação das equipes de saúde bucal na Estratégia Saúde da Família (ESF) ainda é incipiente e não atende às necessidades da população adscrita. ${ }^{25} \mathrm{Em}$ outra pesquisa realizada para analisar as mudanças no trabalho em saúde bucal na atenção primária foi observado que a integralidade é incipiente e com fragilidades a serem enfrentadas no trabalho das equipes da ESF. ${ }^{26}$

Neste cenário de busca pela concretização da universalidade do acesso, da integralidade e da resolutividade da assistência, o SUS está permanentemente se reinventando e uma de suas inovações é o PMAQ-AB que emerge como um facilitador na medida em aponta direcionamentos para o trabalho das equipes de saúde através de 
estratégias de qualificação, acompanhamento e avaliação.

Processos permanentes de avaliação foram institucionalizados com a criação do PMAQ-AB, inserindo uma cultura de monitoramento das informações socioeconômicas, demográficas, epidemiológicas e dos serviços prestados com consequente construção de um diagnóstico situacional do território, essencial para a gestão dos serviços.

O apoio oferecido pelo Ministério da Saúde aos municípios através da instituição de programas de avaliação é fundamental para o avanço do SUS, consolidação da visão ampliada de saúde e fortalecimento do modelo de cuidado integral à saúde.

A territorialização é fortemente valorizada pelo PMAQ-AB que incentiva as equipes a conhecer a população, suas fragilidades, potencialidades, vínculos sociais, perfil de adoecimento e outros determinantes do processo saúde-doença para que possam responder adequadamente às necessidades, organizar agendas, ações programáticas e impactar positivamente na saúde da coletividade.

Travassos et al relatam que a investigação em serviços de saúde assume especial relevância, pois um dos seus objetivos é orientar o desenho de políticas e a melhoria do seu desempenho. ${ }^{27}$

Desta maneira, o PMAQ-AB pretende mensurar os possíveis efeitos da política de saúde com vistas a subsidiar a tomada de decisão, garantir a transparência dos processos de gestão do SUS e dar visibilidade aos resultados alcançados, além de fortalecer o controle social e o foco do sistema de saúde nos usuários. ${ }^{7}$ Ao se investir nestes processos, busca-se contribuir decisivamente com o objetivo de qualificar a atenção à saúde e melhorar o desempenho dos serviços. ${ }^{28}$

O Programa de Melhoria do Acesso e Qualidade da Atenção Básica norteia o desenvolvimento das ações de vigilância em saúde nos municípios brasileiros contribuindo para a estruturação e o fortalecimento do SUS como Política Pública de Saúde.

\section{Considerações finais}


Conclui-se, pelos dados analisados, que a atenção à saúde maternoinfantil mostrou-se bastante resolutiva apresentando altas taxas de vacinação, aleitamento materno exclusivo, captação precoce das gestantes, pré-natal em dia e médias de consultas médicas, com consequente garantia do acesso e da qualidade das ações e serviços, entretanto a atenção em saúde bucal precisa ser aprimorada com a ampliação do acesso às gestantes e aumento da resolutividade dos serviços, especialmente, a conclusão dos tratamentos iniciados.

\section{Referências}

1. Brasil. Lei $n^{\circ} 8.080$, de 19 de setembro de 1990. Dispõe sobre as condições para a promoção, proteção e recuperação da saúde, a organização e o funcionamento dos serviços correspondes e dá outras providencias. Brasília (DF): Diário Oficial da União; 19 set. 1990 [citado em 3 maio 2016]. Disponível em: http://conselho.saude.gov.br/legislacao/lei8080_190990.htm 
2. Ministério da Saúde (BR). PNAB: Política Nacional de Atenção Básica. Brasília (DF): Ministério da Saúde; 2016.

3. Ministério da Saúde (BR). Política Nacional de Humanização: PNH. Brasília (DF): Ministério da Saúde; 2013.

4. Fadel BC, Schneider L, Moimaz SAS, Saliba AN. Administração pública: o pacto pela saúde como uma nova estratégia de racionalização das ações e serviços em saúde no Brasil. RAP. 2009;43(2):445-56. http://dx.doi.org/10.1590/S0034-76122009000200008.

5. Ministério da Saúde (BR). Portaria n 399, de 22 de fevereiro de 2006. Divulga o pacto pela saúde 2006 - consolidação do SUS e aprova as diretrizes operacionais do referido pacto. Brasília, DF: Diário Oficial da União; 22 fev. 2006 [citado em 3 maio 2016]. Disponível em: http://bvsms.saude.gov.br/bvs/saudelegis/gm/2006/prt0399_22_02_2006.html

6. Ministério da Saúde (BR). Portaria $n^{\circ}$ 28, de 8 de janeiro de 2015. Reformula o Programa Nacional de Avaliação de Serviços de Saúde (PNASS). Brasília (DF): Diário Oficial da União; 2015 jan. 8 [citado em 5 maio 2016]. Disponível em: http://bvsms.saude.gov.br/bvs/saudelegis/gm/2015/prt0028_08_01_2015.html

7. Ministério da Saúde (BR). Programa Nacional de Melhoria do Acesso e da Qualidade da Atenção Básica (PMAQ): manual instrutivo. Brasília (DF): Ministério da Saúde; 2015.

8. Ministério da Saúde (BR). Programa Nacional de Melhoria do Acesso e da Qualidade da atenção básica (PMAQ): manual instrutivo. Brasília (DF): Ministério da Saúde; 2012.

9. Secretaria de Estado da Saúde (Rio Grande do Sul). Monitoramento de indicadores de saúde: uma proposta conceitual e metodológica: manual de orientações técnicas. Porto Alegre: Governo do Estado do Rio Grande do Sul; 2007.

10. Magri VL, Aciole GG, Salomão DGF, Tagliaferro SPE, Ribeiro GL. Estudo comparativo de indicadores de saúde bucal em município do estado de São Paulo. Saúde Debate. 2016;40(108):144-55. http://dx.doi.org/10.1590/0103-1104-20161080012.

11. Ministério da Saúde (BR). Portaria $n^{\circ}$ 1.459, de 24 de junho de 2011. Institui, no âmbito do Sistema Único de Saúde - SUS - a Rede Cegonha. Brasília, DF: Diário Oficial da União; 24 jun. 2011 [citado em 3 maio 2016]. Disponível em: http://bvsms.saude.gov.br/bvs/saudelegis/gm/2011/prt1459_24_06_2011.html. 
12. Ministério da Saúde (BR). Portaria $n^{\circ}$ 1.130, de 5 de agosto de 2015. Institui a Política Nacional de Atenção Integral à Saúde da Criança (PNAISC) no âmbito do Sistema Único de Saúde (SUS). Brasília, DF: Diário Oficial da União; 5 ago. 2015 [citado em 3 maio 2016]. Disponível em: http://bvsms.saude.gov.br/bvs/saudelegis/gm/2015/prt1130_05_08_2015.html.

13. Ministério da Saúde (BR). Passo a passo das ações do Brasil Sorridente. Brasília (DF): Ministério da Saúde; 2013.

14. Thomas SL, Wakerman J, Humphreys JS. What core primary health care services should be available to Australians living in rural and remote communities?. BMC Fam Pract. 2014 Aug 21;15:143. http://dx.doi.org/10.1186/1471-2296-15-143.

15. Ministério da Saúde (BR). Nota metodológica da certificação das equipes de Atenção Básica participantes do Programa de Melhoria do Acesso e da Qualidade na Atenção Básica. Brasília (DF): Ministério da Saúde; 2015.

16. Ministério da Saúde (BR). Atenção ao pré-natal de baixo risco. Brasília, DF: Ministério da Saúde; 2012.

17. Costa CRG, Chein MBC, Gama MEA, Coelho LSC, Costa ASV, Cunha CL, et al. Caracterização da cobertura do pré-natal no Estado do Maranhão, Brasil. Bras Enferm. 2010 NovDec;63(6):1005-9. http://dx.doi.org/10.1590/S0034-71672010000600021.

18. World Health Organization (WHO). Antenatal care randomized trial: Manual for the implementation of the new model. Geneva: WHO; 2002.

19. Moimaz SAS, Garbin CAS, Garbin AJI, Zina, LG, Yarid SD, Francisco KMS. Sistema de Informação pré-natal: análise crítica de registros em um município paulista. Rev Bras Enferm. 2010;63(3):385-90. http://dx.doi.org/10.1590/S0034-71672010000300006.

20. Moimaz SAS, Rocha NB, Garbin AJI, Saliba O. Relação entre aleitamento materno e hábitos de sucção não nutritivos. Cienc Saude Colet. 2011;16(5):2477-84. http://dx.doi.org/10.1590/S1413-81232011000500017.

21. Rocha NB, Garbin AJI, Garbin CAS, Saliba O, Moimaz SAS. Estudo longitudinal sobre a prática de aleitamento materno e fatores associados ao desmame precoce. Pesqui Bras Odontopediatria Clin Integr. 2013;13(4):337-42. http://dx.doi.org/10.4034/pboci.2013.134.06. 
22. Maia DR, Sousa FC, Santos IJP, Cavalcante MBC, Nobre NMA. Saúde da criança: análise de indicadores na Atenção Primária. Anais do 120 Congresso de Brasileiro de Medicina de Família e Comunidade; 2013; Belém, PA. Rio de Janeiro: Sociedade Brasileira de Medicina de Família e Comunidade; 2013. 1389 p.

23. Ribeiro LCC, Paula FA, Leite MB, Barbosa BR. As orientações recebidas na estratégia saúde da família e outros serviços de saúde no âmbito da saúde da criança. J Manag Prim Healh Care. 2015;6(1):36-45.

24. Moreira MR, Santin GC, Matos LG, Gravina DBL. Pré-natal odontológico: noções de interesse. J Manag Prim Healh Care. 2015;6(1):77-85.

25. Oliveira JLC, Saliba NA. Atenção odontológica no Programa de Saúde da Família de Campos dos Goytacazes. Cienc Saude Colet. 2005;10(Supl):297-302. http://dx.doi.org/10.1590/S1413-81232005000500030.

26. Scherer CI, Scherer MDA. Advances and challenges in oral health after a decade of the "Smiling Brazil" Program. Rev Saude Publica. 2016;49:98. http://dx.doi.org/10.1590/S00348910.2015049005961.

27. Travassos C, Novaes HMD. Investigação e avaliação em serviços de saúde. Cad Saúde Publica. 2004;20(Supl 2):S144-5. http://dx.doi.org/10.1590/S0102-311X2004000800001.

28. Ministério da Saúde (BR). Análise dos indicadores da Política Nacional de Atenção Básica no Brasil. Brasília (DF): Ministério da Saúde; 2008. 
Suzely Adas Saliba Moimaz | ORCiD: 0000-0002-4949-529X

Doutora em Odontologia Preventiva e Social. Professora do Departamento de Odontologia Infantil e Social da Universidade Estadual Paulista. Araçatuba, SP, Brasil.

Tânia Adas Saliba | ORCiD: 0000-0003-1327-2913

Doutora em Odontologia Legal e Deontologia. Coordenadora do Programa de Pós-Graduação em Odontologia Preventiva e Social da Universidade Estadual Paulista. Araçatuba, SP, Brasil.

\section{Renato Moreira Arcieri}

Doutor em Odontologia. Professor Adjunto do Programa de Pós-Graduação em Odontologia Preventiva e Social da Universidade Estadual Paulista. Araçatuba, SP, Brasil.

Léa Lofego | ORCiD: 0000-0003-0083-3216

Mestranda do curso de Odontologia Preventiva e Social da Universidade Estadual Paulista. Araçatuba, SP, Brasil.

Nemre Adas Saliba | ORCiD: 0000-0001-9608-1631

Doutora em Odontologia Preventiva e Social. Professora Voluntária do Departamento de Odontologia Infantil e Social da Universidade Estadual Paulista. Araçatuba, SP, Brasil. 